\title{
Factors Affecting Early and 1-Year Motor Recovery Following Lumbar Microdiscectomy in Patients with Lumbar Disc Herniation: A Prospective Cohort Review
}

\author{
Vibhu Krishnan Viswanathan, Rajasekaran Shanmuganathan, \\ Siddharth Narasimhan Aiyer, Rishi Kanna, Ajoy Prasad Shetty \\ Department of Spine Surgery, Ganga Hospital, Coimbatore, India
}

\begin{abstract}
Study Design: Prospective cohort study.
Purpose: The study was aimed at evaluating clinicoradiological factors affecting recovery of neurological deficits in cases of lumbar disc herniation (LDH) treated by lumbar microdiscectomy.

Overview of Literature: The majority of the available literature on neurological recovery following neurodeficit is limited to retrospective series. The literature is currently limited regarding variables that can help predict the recovery of neurodeficits following $\mathrm{LDH}$.

Methods: A prospective analysis was performed on 70 consecutive patients who underwent lumbar microdiscectomy (L1-2 to L5-S1) owing to neurological deficits due to LDH. Patients with motor power $\leq 3 / 5$ in L2-S1 myotomes were considered for analysis. Followup was performed at 2, 6, and 12 months to note recovery of motor deficits. Clinicoradiological parameters were compared between the recovered and nonrecovered groups.

Results: A total of 65 patients were available at the final follow-up: 41 (63\%) had completely recovered by 2 months; four showed delayed recovery at the 6-month follow-up; and $20(30.7 \%)$ showed no recovery at 1 year. Clinicoradiological factors, including diabetes, complete initial deficit, areflexia, multilevel disc prolapse, longer duration since initial symptoms, and $\geq 2$ previous symptomatic episodes were associated with a significant risk of poorer recovery ( $p<0.05$ for all). Age, sex, occupation, smoking, level/type or location of disc herniation, primary canal stenosis, disc fragment dimensions, precipitating factors, bladder involvement, bilaterality of symptoms, and the presence or absence of anal reflex did not affect neurological recovery ( $p>0.05$ for all). Diabetes mellitus ( $p=0.033$ ) and complete initial motor deficit $(p=0.028)$ were significantly associated with delayed recovery in the multivariate analysis.

Conclusions: The overall neurological recovery rate in our study was $69 \%$. Diabetes mellitus $(p=0.033)$ and complete initial motor deficit were associated with delayed motor recovery.
\end{abstract}

Keywords: Neurologic recovery; Neurologic deficit; Intervertebral disc displacement; Prognosis; Complication

Received May 13, 2018; Revised Jul 4, 2018; Accepted Jul 26, 2018

Corresponding author: Rajasekaran Shanmuganathan

Department of Spine Surgery, Ganga Hospital, 313, Mettupalayam Road, Coimbatore, Tamil Nadu 641043, India

Tel: +91-9843022325, Fax: +91-422-4383863, E-mail-rajasekaran.orth@gmail.com 


\section{Introduction}

The long-term outcomes for lumbar disc herniation (LDH) are generally considered favorable, and surgery is reserved for indications such as refractory pain, cauda equina syndrome, and progressive neurological deficits [1,2]. Neurological deficits, albeit uncommon, are a significant complication following LDH [3]. The severity of neurological deficits can range from weakness in an isolated myotome to cauda equina syndrome. Neurological recovery in patients with mild neurological deficits (Medical Research Council [MRC] grade $>3 / 5$ ) has been reported to be satisfactory, regardless of the treatment experienced (e.g., conservative vs. surgical treatment) [3]. However, progressive neurological deficits or cauda equina syndrome is considered a surgical emergency, and early surgical decompression has been recommended as the treatment of choice [4-7].

The majority of the available literature on neurological recovery following neurodeficit is limited to retrospective series [8-12]. Neurological recovery following surgical decompression is not universal, and variable recovery rates of between $61 \%$ and $88 \%$ have been reported in the literature [3]. Various factors have been postulated as affecting recovery; however, the contribution of each still remains controversial $[6,9,10]$. Ghahreman et al. [10] in their study observed better neurological recovery following neurodeficit in younger patients, whereas the study by Bhargava et al. [11] observed that the duration and severity of the neurodeficits were significant prognostic indicators for neurological recovery. However, the study by Postacchini et al. [12] failed to reveal any significant prognostic factors. The literature is currently limited to describing variables that can help to predict the recovery of neurodeficits following LDH. The aim of this study was to evaluate neurological motor recovery following lumbar microdiscectomy, and to identify clinicoradiological factors associated with neurological improvement.

\section{Materials and Methods}

The study was a prospective, longitudinal follow-up study that included 70 consecutive patients undergoing microscopic discectomy for motor deficits (with or without associated bladder involvement) following LDH during the period December 2013 to March 2015. The study was approved by the institutional review board of Ganga Hospital, Coimbatore, India (IRB approval no., 04/11/13), and written informed consent was received from all participants prior to study participation.

Patients with motor power $\leq 3 / 5$ (MRC grading: active movement possible against gravity, but not against resistance) in L2-S1 myotomes, with or without bowel bladder deficit, were included in the analysis. Complete motor deficit was defined as motor power of $0 / 5$ in the specific myotome. Sensory loss was sub grouped into complete loss of sensation (S0) and partial loss of sensation (S1). Patients with tandem cervical or thoracic stenosis and isolated sensory deficits were excluded.

\section{Clinical assessment}

Details regarding the patients' general demographic profile, including age, sex, body mass index (BMI), occupation, presence of medical comorbidities, and smoking history were recorded. Patients underwent complete neurological clinical examination (motor, sensory, and autonomic nervous system assessment) preoperatively and postoperatively at the 2, 6, and 12-month followup. At each visit, motor power was assessed by clinical examination based on the MRC power grading system by two independent observers; any disagreement was reviewed by the senior author (R.S.) for the assessment of recovery. The occupational status was defined based on the research study "Physical exertion requirements of work in the dictionary of occupational titles" published by the US Department of Labor [13]. Sedentary and light work were classified as 'light labor' (involving frequent lifting of weights less than $4.5 \mathrm{~kg}$ or lifting of weights of not more than $9.1 \mathrm{~kg}$ at a time, and could involve long hours of standing or walking). Medium, heavy, and very heavy work were classified under 'heavy labor' (involving frequent lifting of weights more than $4.5 \mathrm{~kg}$ or lifting of weights more than $9.1 \mathrm{~kg}$ at a time). 'Current smoker' was defined as a person who has smoked more than 100 cigarettes (or other forms of tobacco that could be smoked) and who has smoked in the past 28 days [14].

The duration of symptoms was classified into three different categories: (1) duration since initial symptoms: the time interval from initial symptoms to performance of surgical decompression for the neurological deficit; (2) onset of current symptomatology: acute $<3$ months of current symptoms, chronic $>3$ months of symptoms, and acute on chronic symptoms, suggested by acute worsening of chronic symptoms; and (3) total number of previous 
symptomatic episodes (number of symptomatic episodes prior to the current episode, with intervening asymptomatic periods). A previous episode was considered significant if the patient reported low back pain accompanied by radiating pain with a dermatomal distribution. Episodes of isolated low back pain without sciatica were excluded.

\section{Radiological assessment}

Radiological assessments, including plain radiographs and magnetic resonance imaging (MRI) scans, were performed for all patients. The radiological factors assessed included the following: (1) number of herniated discs; (2) index level of herniation; (3) nature of herniation (sequestered, extruded, or prolapsed); (4) migration of disc fragment (superior or inferior); (5) herniated disc fragment dimensions; (6) location of herniation on axial section (central, paracentral, foraminal, and posterolateral); (7) percentage of canal compromise on MRI; and (8) bony canal dimensions at the herniated level.

\section{Surgical procedure}

All the patients underwent microlumbar discectomy under general anesthesia. Using either unilateral fenestration alone or wide fenestration, midline laminectomy was performed to access the disc, depending on the location and size of the disc.

\section{Follow-up and assessment}

All the patients were followed up clinically at the end of 2, 6 , and 12 months after discharge. Based on the recovery patterns observed during the follow-up, patients were further classified into recovery and nonrecovery groups. Significant motor recovery was defined as an improvement in motor power to at least MRC grade 4 in the predominantly affected myotomes. All preoperative clinical and radiological factors were studied between the two groups, and a comparison was made to identify any possible associated factors affecting recovery following surgical decompression.

\section{Statistical analysis}

The statistical analysis was performed using IBM SPSS ver. 20.0 (IBM Corp., Armonk, NY, USA). To compare the association of various factors between the recovery and the nonrecovery groups, a univariate analysis using the chi-square test or Fisher's exact test was initially performed. Values with $p<0.05$ were considered significant. We then included only the seven variables that were significant in the univariate analysis (diabetes, longer time interval between first symptoms and surgical decompression, $\geq 2$ previous symptomatic episodes, complete motor deficit, complete sensory loss [S0], areflexia, and number of herniated disc levels $\geq 2$ ) in the multivariate analysis using binary logistic regression. Binary logistic regression is typically used when the response variable is binomial (e.g., yes/no; present/absent) [15]. Due to multicollinearity (a phenomenon in which one variable can be linearly predicted from another predictor variable with a substantial degree of accuracy) between the initial sensory deficit and areflexia, neither of these variables could be considered for the multivariate analysis. Multicollinearity can result in an unstable parameter that can make the assessment in

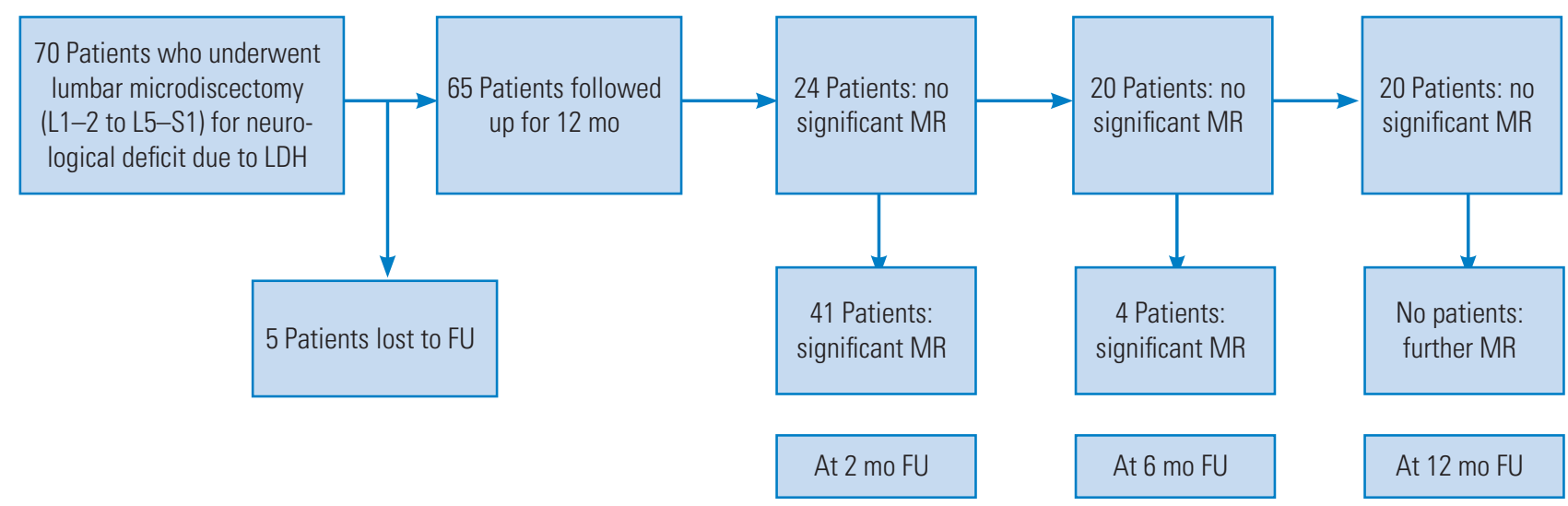

Fig. 1. Flow chart depicting the prospective FU of 70 consecutive patients with motor deficit following LDH. LDH, lumbar disc herniation; FU, followup; MR, motor recovery. 
binary logistic regression of the effect of independent variables on dependent variables difficult [16]. Using binary logistic regression, we determined the impact of each of these seven variables on the odds ratio (OR; exponential of $\beta$ ) of the observed event of interest (i.e., neurological recovery).

\section{Results}

The mean age of the patients in our study was 43.4 years, with 54 men and 16 women. Of the 70 patients, 65 were available for the final follow-up: 41 patients $(63 \%)$ had completely recovered by 2 months; and of the 24 patients who did not recover in the initial 2 months, four (6.1\%) had delayed motor recovery at the 6-month follow-up. These patients showed significant neurological improvement (to a power of at least grade 4 for the predominantly affected myotomes and bladder recovery) at their 6-month follow-up. Twenty patients had not recovered by the end of the 1-year follow-up (Fig. 1). Comparison of clinicoradiological parameters between the sub groups of patients: group 1 (early recovery at 2 months, $\mathrm{n}=41$ ) versus group 2 (late recovery, $\mathrm{n}=4$; or no recovery, $\mathrm{n}=20$ ) are listed in Tables 1-4. Given that the patients with delayed recovery constituted a very small group (only four patients), further analyses were not performed on this subgroup.

\section{Clinical assessment}

Nine of the 24 patients (38\%) in group 2 had diabetes mellitus, whereas only five of the 41 patients (12\%) who recovered had diabetes mellitus ( $p=0.03$; OR, $4.62 ; 95 \%$ confidence interval [CI], 1.31-16.24). Diabetes mellitus was significantly associated with delayed motor recovery, based on both the univariate and multivariate analyses (binary logistic regression analysis: $p=0.033$; OR, 5.683; 95 CI, 1.154-27.980). No other factors concerning the patients' general profile (including age, sex, BMI, occupation, smoking history, and other medical comorbidities) had any significant association with neurological recovery (Tables 1, 4).

Based on the univariate analysis, the patients who recovered had a significantly shorter duration from the initial presenting symptom (mean, 139 days; median, 30 days; range, 2-1,095 days) compared with the patients who did not recover (mean, 349 days; median, 90 days; range, $4-2,555$ days; $p=0.05$ ). However, we could not identify a single best cut-off point for the duration value based on the receiver operating characteristic curve analysis, possibly due to the relatively small sample size. A further binary logistic regression analysis also showed no significant correlation between the time from the initial symptom and recovery from the motor deficit $(p=0.103$; OR, 0.999; 95\% CI, 0.997-1.000).

On the basis of the univariate analysis, the patients who recovered had significantly fewer previous symptomatic episodes (mean, 0.17; median, 0 ; range, $0-2$ ) as compared with the nonrecovered population (mean, 1 ; median, 0 ; range, $0-8 ; p=0.006$; cut-off $\geq 2$ episodes; OR, $14.11 ; 95 \%$ CI, 1.57-127.4). However, in a further logistic regression

Table 1. General profile of patients (group 1 vs. group 2)

\begin{tabular}{|c|c|c|c|c|}
\hline Characteristic & Group $1^{\text {a) }}$ & Group $2^{\text {b) }}$ & $p$-value & $\begin{array}{c}\text { Odds ratio ( } 95 \% \\
\text { confidence interval) }\end{array}$ \\
\hline Age (yr) & $43.15 \pm 2.225(45[17-75])$ & $44.17 \pm 2.311(45[16-66])$ & 0.766 & \\
\hline Sex (male vs. female) & $31: 10$ & 19:5 & 0.935 & \\
\hline Occupation (heavy vs. light labor) & $13: 28$ & $6: 18$ & 0.748 & \\
\hline Smoking history (smokers vs. non-smokers) & $31: 10$ & $17: 7$ & 0.872 & \\
\hline Comorbidity & & & 0.015 & \\
\hline 0 & 34 & 11 & & \\
\hline 1 & 4 & 8 & & \\
\hline 2 & 3 & 5 & & \\
\hline Non-DM vs. DM & $36: 5(12.2)$ & $15: 9(60.0)$ & 0.025 & $4.6(1.31-16.24)$ \\
\hline
\end{tabular}

Values are presented as mean \pm standard deviation (median [range]), number, or number (\%), unless otherwise stated.

DM, diabetes mellitus.

alPatients with neurological recovery. ${ }^{\text {bl }}$ Patients without neurological recovery. 

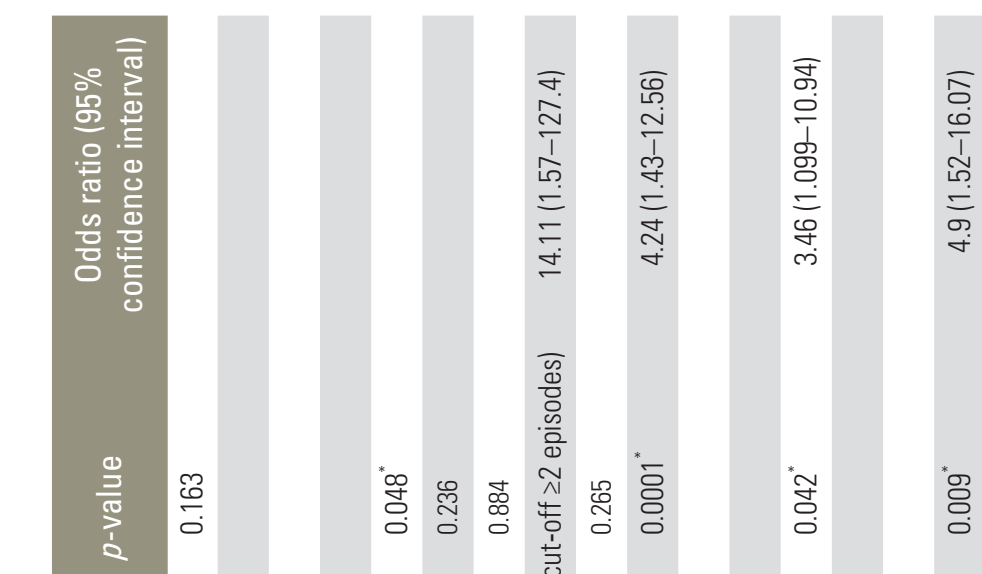

\section{$\frac{\bar{d}}{\bar{\phi}}$}

集兽总

$\stackrel{\infty}{\&}$

‡్丶 :

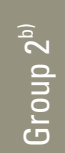

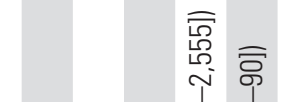

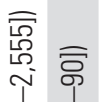

$\therefore=$

足党

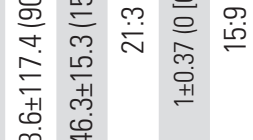

$\left\llcorner\frac{\pi}{\mathscr{d}}\right.$

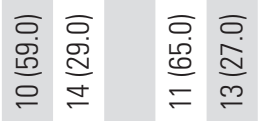

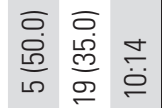

$\widehat{\bar{\sigma}}$

을

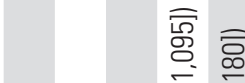

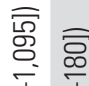

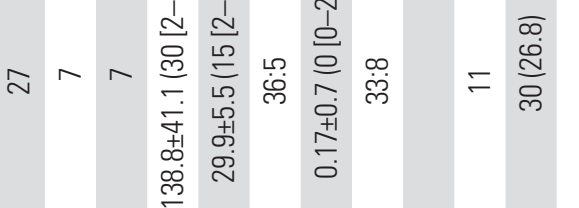

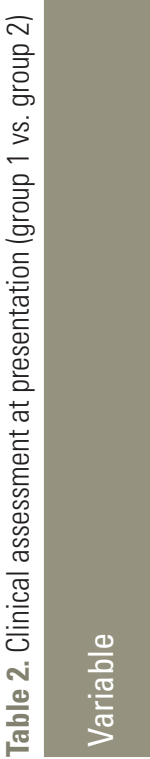

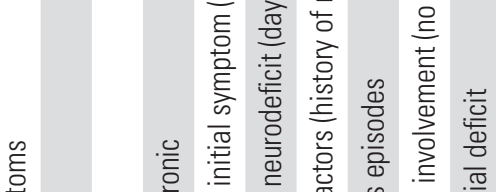

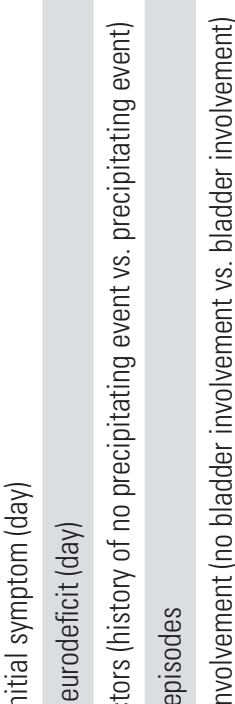

\section{F}

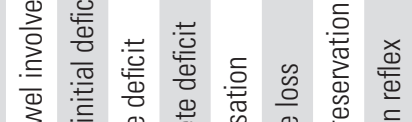

o 0 o $\bar{x}$ o

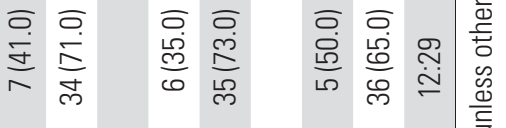

这 वें 
analysis, there was no significant correlation between the number of previous symptomatic episodes and recovery of motor deficits ( $p=0.351$; OR, 3.223; 95\% CI, 0.275-37.789). The duration since the onset of neurological deficits was also not statistically different between the patients who recovered (mean, 30 days; median, 15 days; range, 2-180 days) and those who did not (mean, 46 days; median, 15 days; range, $1-90$ days; $p=0.236$ ).

Only $27 \%$ of the patients who recovered initially had a complete motor deficit. However, $63 \%$ of those who did not recover had a complete initial deficit ( $p=0.0001$; OR, 4.24 ; $95 \%$ CI, 1.43-12.56). In a further logistic regression analysis, there was a significant association between initial completeness of motor deficit and motor recovery ( $p=0.028$; OR, 4.171; 95\% CI, 1.169-14.887). Among the 18 patients who had complete initial sensory loss ( $\mathrm{S} 0$ ), one was lost to follow-up. Of the 17 remaining patients, seven patients (41\%) recovered early, whereas 10 (59\%) did not recover. Among the 48 patients with partial or complete preservation of sensation, 34 (71\%) had recovered early, and only $14(29 \%)$ failed to recover ( $p=0.042$; OR, 3.46; 95\% CI, 1.099-10.94). Similarly, among the 17 patients with areflexia at initial presentation, $11(65 \%)$ did not recover, whereas six (35\%) had an early recovery. Some $73 \%$ of patients ( 35 of 48 ) with preserved reflexes showed recovery ( $p=0.009$; OR, 4.935 ; 95\% CI, 1.52-16.07). Owing to multicollinearity between the initial sensory deficit and areflexia, neither of these variables were considered for multivariate analysis [16]. Other presenting factors, including temporal relation of onset of symptoms $(p=0.16)$, precipitating events $(p=0.88$ ), bilaterality of symptoms $(p=0.412)$, and loss of anal tone $(p=0.48)$, did not differ significantly between the groups.

There were 17 patients with bladder deficit in the cohort. Of the 41 patients in group 1, eight patients presented with coexisting bladder symptoms, whereas the remaining patients with bladder deficits belonged to group 2 . All the patients with poor motor recovery also had some persistent bladder symptoms. However, the difference between these groups with regard to bladder deficit and recovery was not statistically significant $(p=0.265)$. Among the patients with bladder involvement, 10 had decreased anal tone and perianal sensation at presentation. Among the patients with decreased anal tone, 50\% had recovery of bladder function. This difference was not statistically significant $(p=0.480)$ (Table 2).

\section{Radiological assessment}

Thirty-six of the 48 patients with single-disc herniation had a significantly better recovery $(75 \%)$ as compared with five of 17 patients with multilevel herniations (29\%) ( $p=0.006$; OR, 6.6: 95\% CI, 1.9-22.86). In a binary logistic regression analysis, however, no significant correlation was observed between the number of disc herniation levels and motor recovery $(p=0.163$; OR, 0.395 : $95 \% \mathrm{CI}$, $0.108-1.454)$. No other radiological parameters, including index level of herniation, type of herniation (protrusion, extrusion, or sequestration), migration of disc (superior, inferior, or nonmigrated), location of disc (central, paracentral, posterolateral, or foraminal), disc fragment and bony canal dimension, or percentage of spinal canal compromise affected the neurological recovery (Table 3).

Based on the univariate analysis, we identified seven risk factors as poor prognostic variables for an early recovery: diabetes; longer time interval between first symptom and surgical decompression; $\geq 2$ previous symptomatic episodes; complete motor deficit; complete sensory loss (S0); areflexia; and number of herniated disc levels $\geq 2$. The operation characteristics of the estimated univariate model were sensitivity $=75$, specificity $=70.7$, positive likelihood ratio $=2.6$, and negative likelihood ratio $=0.35$. In a further multivariate analysis, only two of these risk factors, diabetes mellitus and completeness of initial motor deficit, could be significantly correlated with the motor recovery after lumbar microdiscectomy.

\section{Discussion}

There is considerable variability in the reported recovery rates for neurodeficit accompanying degenerative lumbar disc disorders. This variability can be ascribed to the heterogenicity in the nature of the underlying pathology, the influence of diverse factors on recovery, the variability in follow-up duration, and the definition of neurodeficit considered during analysis [17]. Age, initial severity of neurodeficit, sensory or autonomic involvement, the duration of the initial deficit or delay in surgical decompression, and comorbid medical illnesses have been postulated to influence neurological recovery [3,10-13,18-21]. However, there is little consensus as to which factors determine prognosis in a patient with neurodeficits [3,10-13,18-21]. The present study was planned to analyze various clinicoradiological factors associated with neurodeficits and their 
Table 3. Radiological assessment (group 1 vs. group 2)

\begin{tabular}{|c|c|c|c|c|}
\hline Variable & Group $1^{\text {a) }}$ & Group $2^{\text {b) }}$ & $p$-value & Odds ratio ( $95 \%$ confidence interval) \\
\hline No. of levels of disc herniation & & & $0.006^{*}$ & $6.6(1.9-22.86)$ \\
\hline 1 & 36 & 12 & & \\
\hline 2 & 5 & 10 & & \\
\hline 3 & 0 & 2 & & \\
\hline Primary level of disc prolapse & & & 0.124 & \\
\hline L1-2 & 0 & 2 & & \\
\hline $\mathrm{L} 2-3$ & 1 & 2 & & \\
\hline Ł3-4 & 8 & 7 & & \\
\hline L4-5 & 30 & 9 & & \\
\hline L5-S1 & 2 & 4 & & \\
\hline Type of disc prolapse & & & 0.722 & \\
\hline Sequestered & 7 & 4 & & \\
\hline Protruded & 7 & 5 & & \\
\hline Extruded & 27 & 15 & & \\
\hline Disc position & & & 0.090 & \\
\hline Inferiorly migrated & 24 & 16 & & \\
\hline Superiorly migrated & 9 & 5 & & \\
\hline Not migrated & 8 & 3 & & \\
\hline Disc location & & & 0.123 & \\
\hline Paracentral & 9 & 6 & & \\
\hline Central & 22 & 14 & & \\
\hline Foraminal & 2 & 0 & & \\
\hline Posterolateral & 8 & 4 & & \\
\hline AP dimension disc (mm) & $8.94 \pm 0.48$ & $8.98 \pm 0.62$ & 0.962 & \\
\hline Canal compromise (\%) & $66.5 \pm 3.4$ & $63.1 \pm 4.4$ & 0.542 & \\
\hline Bony canal dimension (mm) & & & 0.794 & \\
\hline Proximal (AP) & $14.35 \pm 0.35$ & $13.8 \pm 0.49$ & & \\
\hline Proximal (lateral) & $19.82 \pm 0.65$ & $20.08 \pm 0.65$ & & \\
\hline Distal (AP) & $13.85 \pm 0.79$ & $12.38 \pm 0.43$ & & \\
\hline Distal (lateral) & $20.51 \pm 0.83$ & $19.24 \pm 0.72$ & & \\
\hline
\end{tabular}

Values are presented as number, mean \pm standard deviation, unless otherwise stated.

$\mathrm{AP}$, anteroposterior.

${ }^{\text {al }}$ Patients with neurological recovery. ${ }^{\text {bl) }}$ Patients without neurological recovery. $p<0.05$.

influence on motor recovery. This knowledge could help predict neurological recovery among patients undergoing microlumbar discectomy following LDH.

Ghahreman et al. [10] in their study reviewed 56 consecutive patients presenting with ankle dorsiflexion weakness, who showed an overall recovery rate of $41 \%$ with maximal recovery in the first 6 weeks. Among the patients with MRC grade $>3 / 5,68 \%$ showed recovery as compared with $27 \%$ when the MRC power was less than
3/5. Postacchini et al. [12] have prospectively studied 116 patients with neurological deficits and noted a $76 \%$ recovery at a 6-year follow-up. However, they noted persistence of weakness in $39 \%$ of patients with a preoperative MRC grade $\leq 3 / 5$. Our study noted that $63 \%$ of patients recovered in the first 2 months following microlumbar discectomy. A small proportion (6\%) still showed late recovery (at the end of 6 months). Although it is uncommon for patients to recover beyond the initial 2 months, 
Table 4. Binary logistic regression with motor recovery as outcome variable

\begin{tabular}{lcccccc} 
Risk factor & B & Standard error & Wald & $\begin{array}{c}\text { Degree of } \\
\text { freedom }\end{array}$ & $\begin{array}{c}p \text {-value } \\
\text { (95\% confidence interval) }\end{array}$ \\
\hline Diabetes mellitus & 1.737 & 0.813 & 4.565 & 1 & $0.033^{*}$ & $5.683(1.154-27.98)$ \\
\hline Duration since initial symptoms & -0.001 & 0.001 & 2.658 & 1 & 0.103 & $0.999(0.997-1)$ \\
No. of symptomatic episodes & 1.170 & 1.256 & 0.868 & 1 & 0.351 & $3.223(0.275-37.789)$ \\
Completeness of motor deficit & 1.428 & 0.649 & 4.842 & 1 & $0.028^{*}$ & $4.171(1.169-14.887)$ \\
\hline No. of levels of disc herniation & -0.928 & 0.664 & 1.95 & 1 & 0.163 & $0.395(0.108-1.454)$ \\
\hline
\end{tabular}

" $p<0.05$.

there is still a definite possibility of such a recovery even up to 6 months postdecompression. Given that this group was a very small cohort (four patients with late recovery), we could not determine the specific characteristics of this group that showed late recovery. This study made a detailed comparison between patients who recovered early (within 2 months) and those who had a late recovery or no recovery.

Sathian et al. [22] in their study described the pattern of recovery or paresis following a central nervous system injury and observed a fairly predictable time course of recovery in most situations. These researchers concluded that most motor recovery occurred within the first 3 months of the injury, with a stronger recovery happening in the initial 4-8 weeks and reaching a plateau by approximately 12 weeks. Moussellard et al. [23] described the recovery pattern following cervical spondylomyelopathy and observed that the neurological recovery after surgical decompression occurred very rapidly during the first month (statistically significant) and stabilized thereafter (reaching a plateau by 12 months). Dhatt et al. [5] in their study observed a varied pattern of recovery in their group of patients with cauda equina syndrome, with the most common pattern involving motor recovery, followed by bladder and bowel recovery, followed thereafter by sensory recovery. The duration of the recovery in their patients ranged between 4 and 32 months (mean of 13.5 months). Thus, the major portion of the recovery has been shown to occur in the initial postdecompression period. Even in our study, a major proportion of the patients demonstrated neurological recovery at the time of the initial postoperative visit (at 2 months, 41 of 45 patients recovered [91.1\%]), and our statistical analysis was performed for this early recovery group.

Among the various factors relating to the general patient profile, diabetes mellitus was the only factor associ- ated with a delayed recovery on both the univariate and multivariate analyses. Some $38 \%$ of the nonrecovered patients had diabetes, compared with $12.2 \%$ in the recovered population. Diabetes has been associated with overall poorer outcomes in LDH $[24,25]$. The poorer ability of the nerves to heal, along with a possible second hit at a more distal level due to coexisting vasculopathy or neuropathy, could negatively influence the neurological recovery in patients with diabetes [25]. Although certain studies have demonstrated a better recovery in younger patients, this study did not find any association between patient age and recovery of the patient $[10,26]$. A heavy occupational profile has been reported as a possible factor associated with causation of lumbar disc prolapse [27]. However, based on our study, it might not be a significant risk factor in neurological recovery following surgical decompression.

The symptomatology was evaluated under the following categories: (1) onset of symptomatology; (2) time interval between initial presenting symptoms and surgery; (3) number of previous symptomatic episodes; and (4) duration since onset of neurodeficit. In the univariate analysis, a longer time interval from initial symptoms to surgical decompression and number of previous symptomatic episodes $\geq 2$ were significantly associated with a lesser chance of early neurological recovery. However, neither of these factors showed any significant relation to motor recovery in the multivariate (logistic regression) analysis.

Three classic patterns of presentation of cauda equina syndrome have been described: type 1: acute presentation, in which cauda equina syndrome is the first symptom; type 2: culmination of long history of chronic back pain with or without sciatica; and type 3: chronic history with slow progression to numbness and urinary symptoms [6]. Neurological deficits become irreversible when intraspinal pressure passes a critical level for a certain length of time; therefore, chronicity of compression negatively affects the 
recovery [28]. The role of early (6-24 hours) surgery in significantly improving neurological recovery has been discussed in the literature [3,10-12]. In a retrospective analysis by Bhargava et al. [11], the preoperative duration of neurological deficits was demonstrated to be a significant predictor of poor recovery. It has been suggested that surgery could be of questionable benefit in long-standing neurodeficits, given that nerve roots can undergo irreversible changes [3]. Most of our patients generally presented to us quite late (mean, 40.4 days). Even with such delayed presentations, eventual neurological recovery of $69 \%$ was observed. Thus, apart from the delay from onset of neurodeficit, multiple other factors affect the final recovery, and surgery should be offered even in delayed presentations.

The univariate analysis showed that all three components of neurological deficits at presentation (initial complete motor deficit, complete sensory loss, and areflexia) had a significant association with delayed motor recovery. Binary logistic regression analysis also revealed a significant association between initial complete motor deficit and delayed motor recovery. In the review article by Balaji et al. [3], there was no clear evidence from the literature on the association between preoperative severity of deficit and neurological recovery [3]. Although the studies by Ghahrehman et al. [10] and Postacchini et al. [12] have demonstrated a definitive poorer recovery in patients with more severe initial neurodeficits, other studies, such as those by Iizuka et al. [9] and Dubourg et al. [26], have failed to reveal such a significant relationship. It has been previously demonstrated that complete sensory loss is a poor prognostic factor for traumatic spinal cord injuries [28]. We observed that complete, initial motor deficit negatively influenced the neurological outcome in our patients (based on both the univariate and multivariate analyses).

Among the 17 patients with initial bladder deficits, $47 \%$ had good early motor recovery, whereas $53 \%$ failed to recover. Although there was slight tendency toward poorer motor recovery in patients with initial bladder involvement, the difference was not statistically significant ( $p=0.265$ ). Among patients with bladder involvement, no significant association was found between decreased initial anal tone/reflexes and motor or bladder recovery $(p>0.05)$. Previous studies in the literature have demonstrated poorer neurological outcomes in patients with significant bladder function involvement [5,7]. Cauda equina syndrome (CES) has been classified into two stages based on bladder involvement: CES-incomplete (CESI); and CES with true retention (CES-R) [6]. It has been emphasized that CES-R (with bladder retention) leads to poorer outcomes as compared with the CES-I stage, and timely intervention at CES-I stage might halt further progression into its more irreversible stage. Dhatt et al. [5] in their study have demonstrated that the absence of anal reflex at presentation is a predictor of poor recovery in patients with cauda equina syndrome.Although the univariate analysis revealed that multilevel disc prolapses had a poorer recovery even after decompression, the multivariate analysis of the binary regression model failed to reveal such an outcome. A multilevel insult has been shown to cause significant damage to neural elements (double-crush phenomenon) in such a way that recovery can become more compromised than recovery in a single-level pathology [19]. No other radiological factors, including disc morphology, canal dimensions, and disc dimension, affected neurological recovery.

Among a group of patients with severe neurodeficits, Postacchini et al. [12] have noted that those who underwent complete recovery had a relatively earlier surgical decompression as compared with the patients with persistent weakness ( 35 days versus 69 days). Conversely, Balaji et al. [3], in a systematic review of severe neurodeficit recovery, have concluded that there is insufficient evidence to suggest that early surgery could improve recovery. The authors have also noted that a majority of the studies analyzing neurological recovery have not included a multiple regression analysis, which is a significant limitation. Macki et al. [29] retrospectively reviewed 71 patients undergoing decompression for foot drop using a discrete time proportional hazards model, and analyzed recovery at six different time intervals. The authors noted that preoperative muscle strength and duration of weakness were significant predictors of recovery from foot drop. Overdevest et al. [30] in their study performed a randomized controlled trial, comparing recovery in 150 patients with neurological deficits accompanying LDH. They reported comparable overall recovery for both the surgical and the conservative treatment groups, with a faster recovery favoring surgery in the early follow-up. This difference was not observed at the 26- and 52-week follow-ups.

\section{Conclusions}

Our study identified a set of seven risk factors (on the 
basis of a univariate analysis) that could predict poor recovery in patients with motor deficits following LDH: diabetes; longer time interval between the occurrence of the first symptom and surgical decompression; $\geq 2$ previous symptomatic episodes; complete motor deficit; complete sensory loss (S0); areflexia; and number of herniated disc levels $\geq 2$. On the basis of a binary logistic regression analysis, only diabetes mellitus and complete initial motor deficit revealed a significant association with delayed motor recovery.

There are some limitations to this study. First, the sample size of our study population was small. We unfortunately did not perform a power analysis prior to recruiting the patients for the study to identify the ideal size for the required sample population. Secondly, there is a possibility that factors such as duration of symptoms and the time from the neurological deficit might not be accurate, due to associated recall bias. Thirdly, the surgical intervention could have been delayed in certain patients, due to a possible delay in referral or a lack of patient awareness/inclination toward surgery (which are known factors influencing patient management in developing parts of the world). A larger, randomized controlled study can not only help us better understand the influence of these clinicoradiological factors on neurological recovery, but could also help us identify the best subset of combined predictors that might exert the greatest influence on neurological recovery. We nevertheless believe that the observations from our current study provide insight into the clinical and radiological factors that can influence motor recovery and thereby help us in predicting outcome following microlumbar discectomy.

\section{Conflict of Interest}

No potential conflict of interest relevant to this article was reported.

\section{Acknowledgments}

This study was funded by Ganga Orthopaedic Research \& Education Foundation, Coimbatore, India.

\section{References}

1. Peul WC, van Houwelingen HC, van den Hout WB, et al. Surgery versus prolonged conservative treatment for sciatica. N Engl J Med 2007;356:2245-56.

2. Jacobs WC, van Tulder M, Arts M, et al. Surgery versus conservative management of sciatica due to a lumbar herniated disc: a systematic review. Eur Spine J 2011;20:513-22.

3. Balaji VR, Chin KF, Tucker S, Wilson LF, Casey AT. Recovery of severe motor deficit secondary to herniated lumbar disc prolapse: is surgical intervention important?: a systematic review. Eur Spine J 2014;23:1968-77.

4. Korse NS, Pijpers JA, van Zwet E, Elzevier HW, Vleggeert-Lankamp CL. Cauda Equina Syndrome: presentation, outcome, and predictors with focus on micturition, defecation, and sexual dysfunction. Eur Spine J 2017;26:894-904.

5. Dhatt S, Tahasildar N, Tripathy SK, Bahadur R, Dhillon M. Outcome of spinal decompression in Cauda equina syndrome presenting late in developing countries: case series of 50 cases. Eur Spine J 2011;20:2235-9.

6. Gitelman A, Hishmeh S, Morelli BN, et al. Cauda equina syndrome: a comprehensive review. Am J Orthop (Belle Mead NJ) 2008;37:556-62.

7. Srikandarajah N, Boissaud-Cooke MA, Clark S, Wilby MJ. Does early surgical decompression in cauda equina syndrome improve bladder outcome? Spine (Phila Pa 1976) 2015;40:580-3.

8. Kostuik JP. Medicolegal consequences of cauda equina syndrome: an overview. Neurosurg Focus 2004;16:e8.

9. Iizuka $Y$, Iizuka $H$, Tsutsumi $S$, et al. Foot drop due to lumbar degenerative conditions: mechanism and prognostic factors in herniated nucleus pulposus and lumbar spinal stenosis. J Neurosurg Spine 2009;10:260-4.

10. Ghahreman A, Ferch RD, Rao P, Chandran N, Shadbolt $\mathrm{B}$. Recovery of ankle dorsiflexion weakness following lumbar decompressive surgery. J Clin Neurosci 2009;16:1024-7.

11. Bhargava D, Sinha P, Odak S, Tyagi A, Towns G, Pal D. Surgical outcome for foot drop in lumbar degenerative disease. Global Spine J 2012;2:125-8.

12. Postacchini F, Giannicola G, Cinotti G. Recovery of motor deficits after microdiscectomy for lumbar disc herniation. J Bone Joint Surg Br 2002;84:1040-5.

13. Social Security Administration. Code of Federal Reg- 
ulations: 404.1567: physical exertion requirements [Internet]. Woodlawn (MD): Social Security Administration [cited 2018 Apr 24]. Available from: https:// www.ssa.gov/OP_Home/cfr20/404/404-1567.htm.

14. Ryan H, Trosclair A, Gfroerer J. Adult current smoking: differences in definitions and prevalence eestimates: NHIS and NSDUH, 2008. J Environ Public Health 2012;2012:918368.

15. Sperandei S. Understanding logistic regression analysis. Biochem Med (Zagreb) 2014;24:12-8.

16. Kumar TK. Multicollinearity in regression analysis. Rev Econ Stat 1975;57:365-6.

17. Wang Y, Nataraj A. Foot drop resulting from degenerative lumbar spinal diseases: clinical characteristics and prognosis. Clin Neurol Neurosurg 2014;117:339.

18. Cribb GL, Jaffray DC, Cassar-Pullicino VN. Observations on the natural history of massive lumbar disc herniation. J Bone Joint Surg Br 2007;89:782-4.

19. Girardi FP, Cammisa FP Jr, Huang RC, Parvataneni $\mathrm{HK}$, Tsairis P. Improvement of preoperative foot drop after lumbar surgery. J Spinal Disord Tech 2002;15:490-4.

20. Aono H, Nagamoto Y, Tobimatsu H, Takenaka S, Iwasaki M. Surgical outcomes for painless drop foot due to degenerative lumbar disorders. J Spinal Disord Tech 2014;27:E258-61.

21. Liu K, Zhu W, Shi J, et al. Foot drop caused by lumbar degenerative disease: clinical features, prognostic factors of surgical outcome and clinical stage. PLoS One 2013;8:e80375.

22. Sathian K, Buxbaum LJ, Cohen LG, et al. Neurological principles and rehabilitation of action disorders: common clinical deficits. Neurorehabil Neural Repair 2011;25(5 Suppl):21S-32S.
23. Moussellard HP, Meyer A, Biot D, Khiami F, Sariali E. Early neurological recovery course after surgical treatment of cervical spondylotic myelopathy: a prospective study with 2-year follow-up using three different functional assessment tests. Eur Spine J 2014;23:1508-14.

24. Sekiguchi M, Konno S, Anzai H, Kikuchi S. Nerve vasculature changes induced by serotonin under chronic cauda equina compression. Spine (Phila Pa 1976) 2002;27:1634-9.

25. Sakellaridis N. The influence of diabetes mellitus on lumbar intervertebral disk herniation. Surg Neurol 2006;66:152-4.

26. Dubourg G, Rozenberg S, Fautrel B, et al. A pilot study on the recovery from paresis after lumbar disc herniation. Spine (Phila Pa 1976) 2002;27:1426-31.

27. Mundt DJ, Kelsey JL, Golden AL, et al. An epidemiologic study of non-occupational lifting as a risk factor for herniated lumbar intervertebral disc: the Northeast Collaborative Group on Low Back Pain. Spine (Phila Pa 1976) 1993;18:595-602.

28. Zariffa J, Kramer JL, Fawcett JW, et al. Characterization of neurological recovery following traumatic sensorimotor complete thoracic spinal cord injury. Spinal Cord 2011;49:463-71.

29. Macki M, Syeda S, Kerezoudis P, Gokaslan ZL, Bydon A, Bydon M. Preoperative motor strength and time to surgery are the most important predictors of improvement in foot drop due to degenerative lumbar disease. J Neurol Sci 2016;361:133-6.

30. Overdevest GM, Vleggeert-Lankamp CL, Jacobs WC, et al. Recovery of motor deficit accompanying sciatica: subgroup analysis of a randomized controlled trial. Spine J 2014;14:1817-24. 